\title{
X-ray spectroscopic study of the electronic structure of the high-dielectric-constant material $\mathrm{CaCu}_{3} \mathrm{Ti}_{4} \mathrm{O}_{12}$
}

\author{
Cormac McGuinness \\ Department of Physics, Trinity College Dublin, Dublin 2, Ireland \\ James E. Downes, ${ }^{*}$ Paul Sheridan, ${ }^{\dagger}$ P.-A. Glans, and Kevin E. Smith \\ Department of Physics, Boston University, 590 Commonwealth Avenue, Boston, Massachusetts 02215, USA \\ W. Si and Peter D. Johnson \\ Department of Physics, Brookhaven National Laboratory, Upton, New York 11973, USA \\ (Received 13 December 2004; revised manuscript received 1 March 2005; published 23 May 2005)
}

\begin{abstract}
The element-specific valence- and conduction-band densities of states for the high-dielectric-material $\mathrm{CaCu}_{3} \mathrm{Ti}_{4} \mathrm{O}_{12}$ have been measured using soft x-ray emission and absorption spectroscopies. Ti $L_{\alpha, \beta}, \mathrm{Cu} L_{\alpha, \beta}$, and $\mathrm{O} K$ soft x-ray emission spectra of $\mathrm{CaCu}_{3} \mathrm{Ti}_{4} \mathrm{O}_{12}$ were measured with monochromatic photon excitation on selected energies above the $\mathrm{Ti}$ and $\mathrm{Cu} L_{2,3}$ and $\mathrm{O} K$ absorption edges, respectively. X-ray absorption spectra were recorded at the same edges. The electronic structure was also calculated using density functional theory employing the full-potential linearized augmented plane-wave method. Excellent agreement is seen between the results of these calculations and the measured x-ray emission and absorption spectra. This agreement is particularly good at the $\mathrm{O} K$ edge where the resonant behavior of the x-ray emission spectrum can be attributed directly to $\sigma$ - and $\pi$-state emission from valence-band $\mathrm{O} 2 p$ states when in resonance with $\pi^{*}$ and $\sigma^{*}$ conduction-band $\mathrm{O} 2 p$ states. Resonant inelastic x-ray scattering is observed at the $\operatorname{Ti} L_{2,3}$ absorption edge and is compared to previous studies of Ti containing perovskite compounds. The role of $\mathrm{Cu} 3 d$ states in determining the band gap of this material is discussed.
\end{abstract}

DOI: 10.1103/PhysRevB.71.195111

PACS number(s): 78.70.En, 77.55.+f, 71.20.Ps

\section{INTRODUCTION}

There is significant technological interest in the development of new materials with a relatively temperature independent high static dielectric constant $\left(\varepsilon_{0}\right)$ for use in microelectronic devices. One possible candidate material that has attracted much recent attention is $\mathrm{CaCu}_{3} \mathrm{Ti}_{4} \mathrm{O}_{12}$ (CCTO). ${ }^{1-3}$ Single crystals of this material display an exceptionally high $\varepsilon$ of $8 \times 10^{4}$ at room temperature, and $\varepsilon_{0}$ remains essentially constant from $100 \mathrm{~K}$ to $600 \mathrm{~K} .{ }^{1,2}$ Thin films of CCTO have recently been synthesized using pulsed laser deposition, and these films show a temperature independent $\varepsilon_{0}$ of approximately $1.5 \times 10^{3}$, above $100-150 \mathrm{~K}^{4}$

Much remains unknown about the basic physics of CCTO. First, the dielectric constant of the single crystals drops by a factor of $10^{3}$ when cooled below $100 \mathrm{~K} .{ }^{1-3}$ Second, while a high $\varepsilon_{0}$ is often associated with either a ferroelectric material (with a permanent electric dipole moment) or a relaxor material, CCTO does not display a ferroelectric lattice distortion in either x-ray or neutron diffraction. ${ }^{1,2}$ Recent work points towards the possibility that the giant $\varepsilon_{0}$ is partially due to internal barrier layer capacitance resulting from heavy twinning in the crystals. ${ }^{1,5,6}$ It has also been suggested that the material contains regions that are either insulating or semiconducting. ${ }^{6}$ However, to date there have been no detailed spectroscopic measurements of the valence- and conduction-band electronic structure of CCTO.

We present here the results of a soft x-ray emission (SXE) spectroscopy and soft $\mathrm{x}$-ray absorption spectroscopy (XAS) study of the electronic structure of thin-film $\mathrm{CaCu}_{3} \mathrm{Ti}_{4} \mathrm{O}_{12}$ at room temperature. These are bulk probes of the electronic structure $^{7}$ and, in contrast to photoemission spectroscopy, can be applied with ease to insulating samples such as high dielectric materials. ${ }^{8}$ We have measured the elementresolved partial density of states for electrons associated with $\mathrm{Ti}, \mathrm{O}$, and $\mathrm{Cu}$ atoms in CCTO. We have also observed resonant inelastic $\mathrm{x}$-ray scattering features in the emission spectra that are associated with excitations across the band gap. Our density-of-states measurements are compared directly to theory.

\section{EXPERIMENTAL METHOD}

The experiments reported here were undertaken on soft X-ray undulator beamline X1B at the National Synchrotron Light Source (NSLS) at Brookhaven National Laboratory. This beamline is equipped with a spherical grating monochromator. Soft x-ray emission spectra were recorded using a Nordgren-type grazing-incidence spherical grating spectrometer with a total energy resolution of $\sim 0.4 \mathrm{eV}$ at the $\mathrm{O} \mathrm{K}$ edge. ${ }^{7,9}$ The resolution of the monochromator for XAS measurements was set to 0.10 and $0.13 \mathrm{eV}$ at the Ti $L$ edge and $\mathrm{O} K$ edge, respectively. XAS spectra were recorded both by the sample drain current technique to obtain the total electron yield (TEY) and by measuring the total fluorescence yield (TFY) with normal incidence of the incoming radiation. For SXE measurements the resolution of the monochromator was set to $\sim 0.83(0.77) \mathrm{eV}$ at the Ti $L$ edge (O $K$ edge) with the spectrometer resolution set at $\sim 0.57(0.53) \mathrm{eV}$ leading to a combined spectrometer-monochromator resolution of 1.00 
(0.94) $\mathrm{eV}$ for the resonant SXE spectra presented here. In order to obtain reasonable statistical accuracy in the SXE spectra the data were acquired for $60-120$ min per spectrum. The energy scale of the monochromator was calibrated at the Ti $L$ edge and $\mathrm{O} K$ edge by comparison of the XAS spectra of rutile $\mathrm{TiO}_{2}$ to previously published results. ${ }^{10}$ The energy scales of the recorded emission spectra at the Ti $L$ edge and $\mathrm{O} K$ edge were calibrated by reference to the known peak emission energies of $\mathrm{Cu}$ and $\mathrm{Zn}$ metals, respectively, both observed in second order. All measurements were performed with the samples at room temperature. Vacuum pressure during the measurements was below $5 \times 10^{-9}$ Torr. The $\mathrm{CaCu}_{3} \mathrm{Ti}_{4} \mathrm{O}_{12}$ samples were in the form of epitaxial thin films produced by pulsed laser deposition onto $\mathrm{LaAlO}_{3}$ substrates. ${ }^{4}$ The thickness of the films was $1.2 \mu \mathrm{m}$.

The shape of observed x-ray emission spectra can be affected by self-absorption where there is an overlap in energy between the features in the emission spectra and the absorption spectrum. This is particularly the case for excitation energies above the transition-metal $L_{2}$ edges. A correction factor can be given by:

$$
I_{\text {corrected }}=I_{\text {measured }}\left[1+\frac{\mu_{o}}{\mu_{i}} \tan \left(\theta_{i}\right)\right],
$$

where $\theta_{i}$ is the grazing angle of incidence of the incoming radiation onto the sample, and $\mu_{i}$ and $\mu_{o}$ are the absorption coefficients for the incoming and outgoing photons, respectively. ${ }^{11,12}$ The surface of the sample is assumed to be flat, and in our measurement geometry the incident and outgoing photon directions are at $90^{\circ}$ to each other. The measured transition-metal edge $L_{2,3}$ absorption spectrum can then be normalized to match the known values of the atomic photoabsorption cross section above and below the transition-metal edge. ${ }^{13}$ The derived values of $\mu_{i}$ and $\mu_{o}$ may then be used to correct the measured emission spectrum, taking into account the instrumental resolutions used in measuring the emission spectrum. In our measurements with $\theta_{i}$ $\sim 20^{\circ}$ the correction factor is less than $2 \%$ for the Ti $L_{3}$ emission features and can be up to $40 \%$ for the Ti $L_{2}$ emission peak for comparable excitation energies. At excitation energies above the main absorption features the selfabsorption losses increase and the correction factor can be $>2$. All transition-metal $L$ edge spectra presented here have the correction factor applied.

\section{RESULTS: THEORY AND EXPERIMENT}

As XAS and SXE spectroscopy probe the unoccupied and occupied partial densities of states (PDOS) respectively, it is worthwhile to compare the measured spectra with the results of density-of-states calculations. Several band-structure calculations for CCTO exist in the literature, and in particular He et al. have calculated the element specific PDOS. ${ }^{14}$ However, their report presents the PDOS only for a very narrow energy range around the Fermi level, $E_{\mathrm{F}} .{ }^{14}$ In order to make a full comparison to our measurements, we performed a new calculation based on density functional theory (DFT). The DFT calculations presented here employ the full-potential linearized augmented plane-wave (FP-LAPW) method as

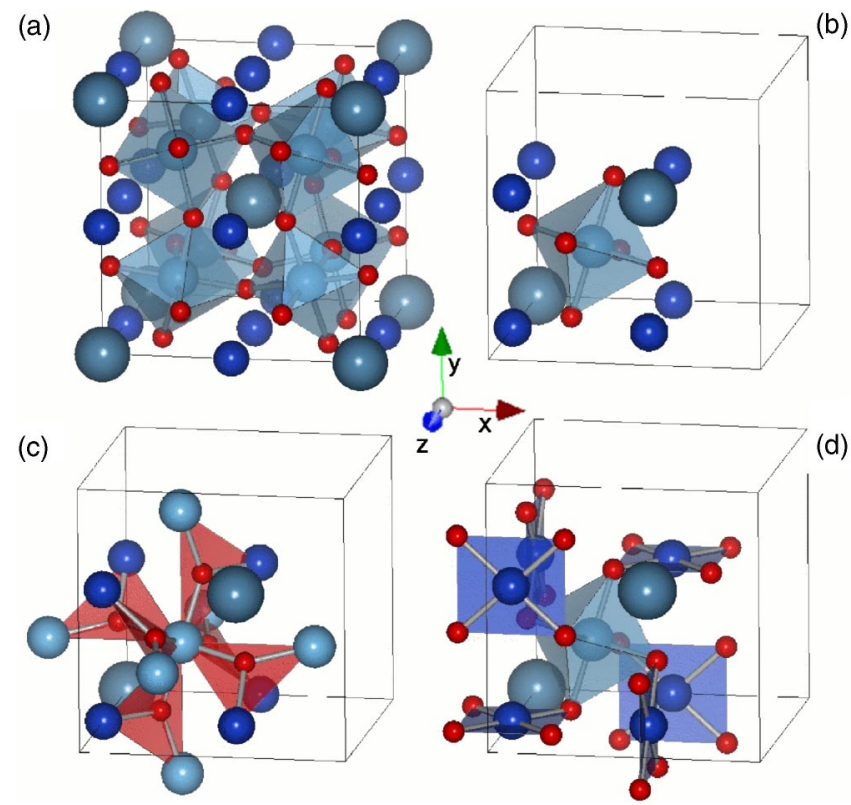

FIG. 1. (Color online) The conventional cubic cell showing the crystal structure of CCTO with (a) all atoms in the conventional cubic cell comprising two formula units, (b) displaying 1/8 of the cell showing the octahedral coordination of the Ti atom, (c) showing the local planar coordination of each $\mathrm{O}$ atom, and (d) showing the planar coordination of each $\mathrm{Cu}$ atom.

implemented in the WIEN $2 \mathrm{k}$ code. ${ }^{15}$ The recommended standard settings of the code were used as well as the generalized gradient approximation (GGA) of Perdew et al. ${ }^{16}$ The radius of the muffin tin spheres was $1.0,1.0,1.0$, and $0.95 \AA$ for $\mathrm{Ca}, \mathrm{Cu}, \mathrm{Ti}$, and $\mathrm{O}$, respectively. The Brillouin zone was sampled with $100 k$ points in order to obtain the DOS and PDOS results presented here. The electronic structure was computed using the observed crystal structure (space group Im3) and the experimentally determined lattice constant of $7.384 \AA$ as inputs. Figure 1 presents a diagram of the crystal structure of CCTO, displaying the conventional cubic unit cell with eight $\mathrm{TiO}_{6}$ octahedra as well as the local coordination of the $\mathrm{Ti}, \mathrm{O}$, and $\mathrm{Cu}$ atoms. Titanium is octahedrally coordinated, and the oxygen atoms are in a planar trigonal arrangement while the copper atoms are at the center of rectangular $\mathrm{CuO}_{4}$ plaquettes which are mutually isolated from each other. Figure 2 presents the total DOS as well as the spin components of the $\mathrm{Cu} 3 d$, Ti $3 d$, and O $2 p$ PDOS. The overall DOS agrees very well with the calculations of He et $a l .{ }^{14}$ except for slight differences in the positioning and distribution of the states just above and below $E_{\mathrm{F}}$ which are principally due to $3 d$ orbitals localized on the $\mathrm{Cu}^{2+}$ ions. These are identified as $\mathrm{Cu} 3 d_{x y}$ states and the calculated gap is due to the separation between the spin components of these states. ${ }^{14}$ This gap is much smaller than that estimated from optical conductivity measurements of $1.5 \mathrm{eV} .^{3}$ Similarly to $\mathrm{He}$ et $a l .{ }^{14}$ the conduction band exhibits a pronounced double-peaked structure, with the primary features in the unoccupied PDOS separated by approximately $3 \mathrm{eV}$. The conduction band is dominated by the Ti $3 d$ states, crystal field split into $t_{2 g}$ and $e_{g}$ components, while the valence band is dominated by $\mathrm{O} 2 p$ states and $\mathrm{Cu} 3 d$ states. From Fig. 2 


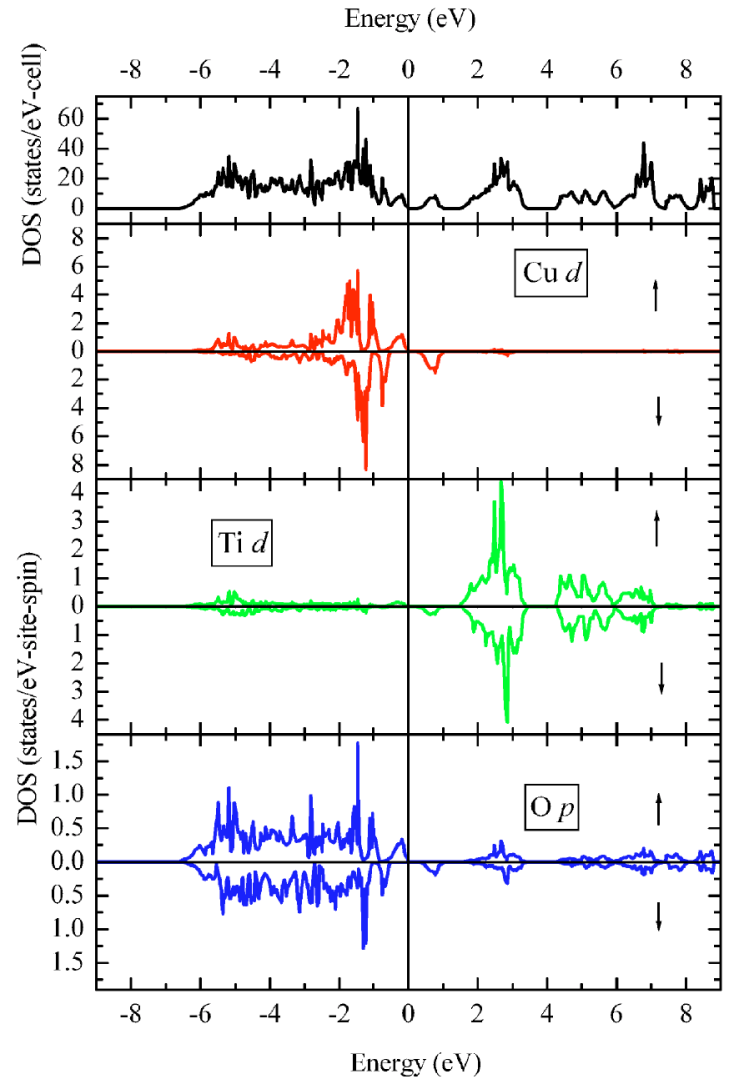

FIG. 2. Total DOS (states/eV cell spin) for CCTO and site- and angular-momentum-projected PDOS (states/eV site spin) for $\mathrm{Cu} d$, Ti $d$, and $\mathrm{O} p$ states. Arrows indicate direction of spin while the vertical line indicates the valence-band maximum. The Ca $d$ PDOS is negligible except above an onset of $6.5 \mathrm{eV}$ with a large peak just above that energy.

there is also a contribution to the valence band due to Ti $3 d$ states especially considering from the expected formal valence states of the constituents that this is nominally a Ti $d^{0}$ compound. The strong $\mathrm{O} 2 p$ and $\mathrm{Cu} 3 d$ hybridization indicates that there is a highly covalent $\mathrm{Cu}-\mathrm{O}$ bond. Charge transfer excitations such as $\mathrm{O} 2 p \rightarrow \mathrm{Ti} 3 d$ play a large role in the metal-edge SXE spectra in CCTO.

\section{A. Titanium states}

Figure 3 presents the Ti $L_{2,3}$ SXE and XAS spectra of $\mathrm{CaCu}_{3} \mathrm{Ti}_{4} \mathrm{O}_{12}$. The Ti $L_{2,3}$-edge XAS spectrum of is shown at the right of Fig. 3 and is measured in the TEY mode. The main features of the XAS spectrum are spin split into the $L_{3}$ and the $L_{2}$ components. The separation between the $L_{2}$ and $L_{3}$ edges is measured at $5.5 \mathrm{eV}$ in close agreement with $\mathrm{x}$-ray photoemission spectroscopy (XPS) measurements of the Ti $2 p$ spin-orbit core-level splitting of $5.6 \mathrm{eV}$ in $\mathrm{BaTiO}_{3} .{ }^{17}$ The SXE spectrum on the left of the figure was recorded with an incident photon energy of $460.9 \mathrm{eV}$, below the $L_{2}$ edge and corresponding to the second large peak in the XAS spectrum. At this energy, only electrons from Ti $2 p_{3 / 2}$ states are excited, so the SXE spectrum results from the deexcitation of electrons with Ti $3 d$ character into the Ti $2 p_{3 / 2}$ hole. The Ti $L_{2,3}$

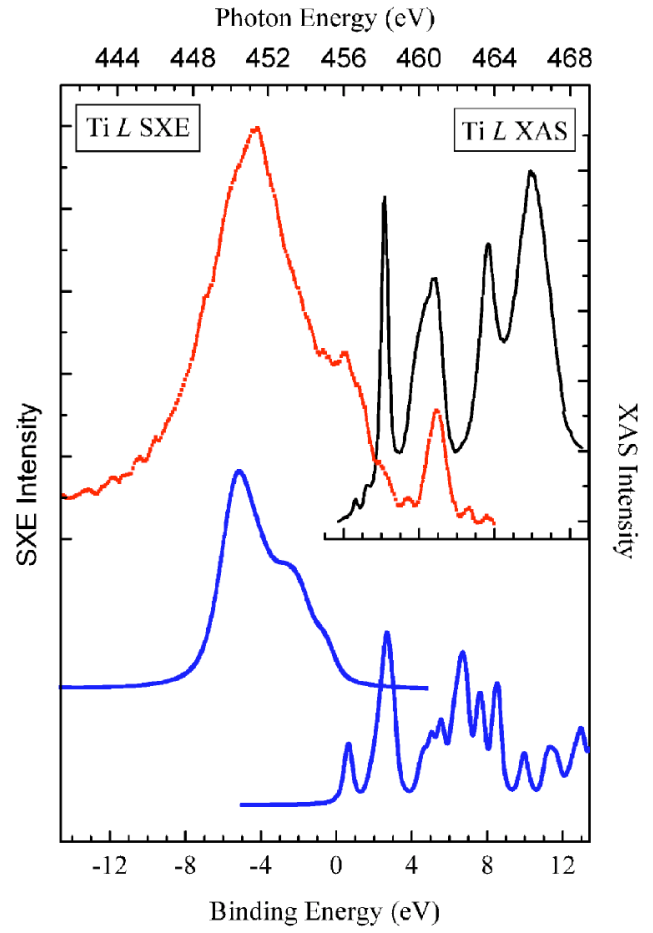

FIG. 3. Ti $L$-edge XAS and SXE spectra from CCTO are shown in the upper part of the figure. The XAS spectrum on the right of the figure corresponds to absorption from the Ti $2 p_{1 / 2}$ and $2 p_{3 / 2}$ states. The SXE spectrum on the left was recorded with an excitation energy of $460.9 \mathrm{eV}$ and corresponds to radiative transitions into holes on the Ti $2 p_{3 / 2}$ level. On the lower half of the figure a model SXE spectrum is shown at the left based upon the calculated valence-band Ti $3 d$ and $4 s$ PDOS. At the lower right is the Ti $3 d$ conduction band PDOS. The calculated SXE spectrum and calculated conduction-band PDOS are both broadened by appropriate lifetime and instrumental effects. Spectra and calculations are vertically offset for clarity. Experimental and theoretical energy scales are aligned by reference to the $t_{2 g}$ peak in the XAS spectrum.

XAS spectrum for CCTO is similar to that measured in other titanium oxide perovskites such as $\mathrm{SrTiO}_{3}, \mathrm{BaTiO}_{3}$, and $\mathrm{Ba}_{0.5} \mathrm{Sr}_{0.5} \mathrm{TiO}_{3} .{ }^{18}$ Four distinct absorption features are visible, as well as two small prepeak features at lower energies. As the dipole-allowed transitions for $2 p$ XAS are $2 p \rightarrow 3 d$ and $2 p \rightarrow 4 s$ (with a higher probability for the $2 p \rightarrow 3 d$ transitions), it is tempting to compare the measured absorption spectrum to the calculated Ti $3 d$ DOS for the conduction band. However, some caution is required in this comparison since the DOS calculated by the DFT method above is obtained in a single-particle approximation and does not include the $3 d-3 d$ and $2 p-3 d$ two particle interactions necessary to accurately reproduce the spectral shape of the Ti $L$ edge XAS. ${ }^{19,20}$ Further the conduction-band DOS as calculated by DFT in this instance is a ground-state calculation and the empty states are virtual states and not excited states. In common with many other compounds containing $\mathrm{Ti}$ at octahedral sites the Ti $L$-edge XAS exhibits small leading peaks which may be modeled accurately through application of atomic multiplet calculations which include the appropriate crystal-field splitting. ${ }^{20}$ The first main peak at $458.2 \mathrm{eV}$ is a $2 p_{3 / 2}$ transition to a $t_{2 g}^{*}$ final state while that at 


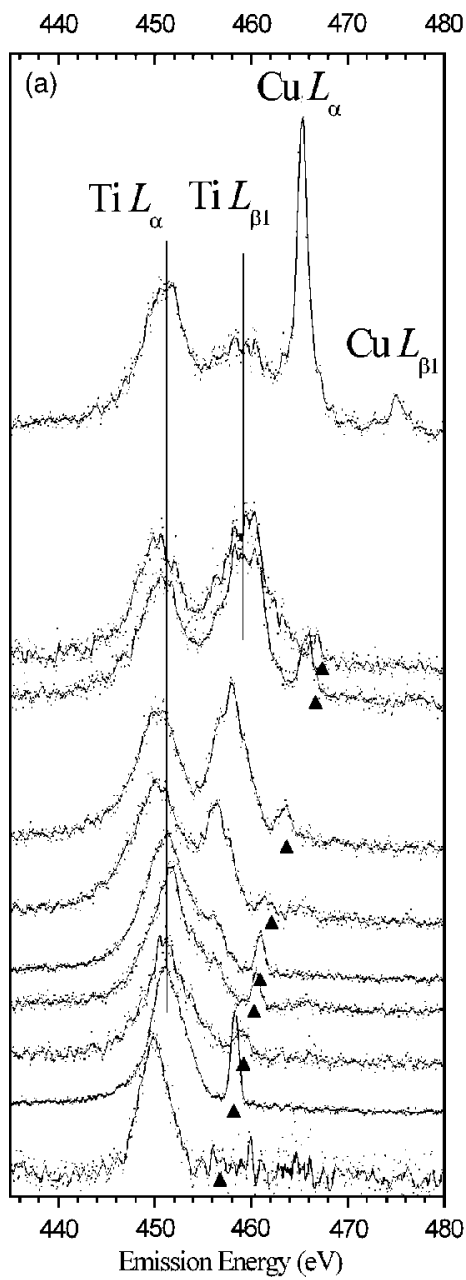

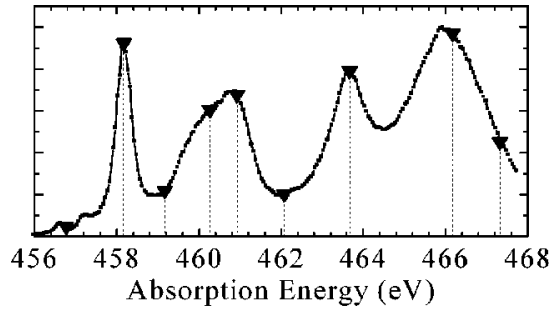

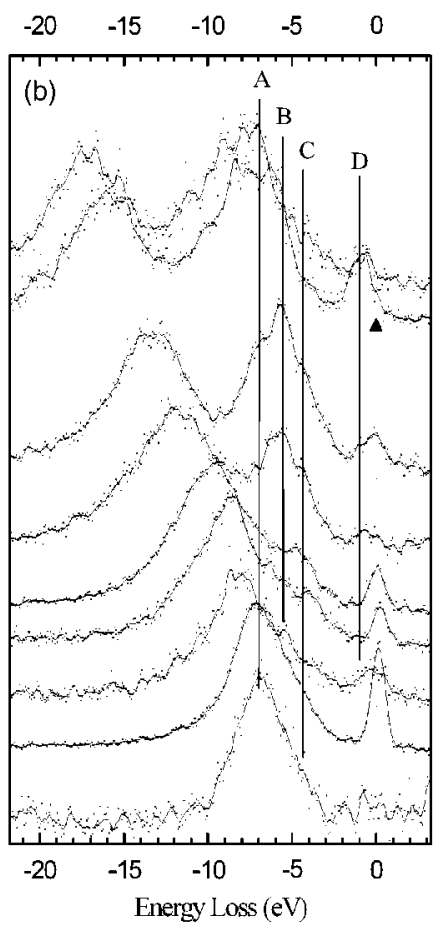

FIG. 4. (a): Series of Ti $L$-edge SXE spectra as a function of excitation energy. (b) The same series of Ti $L$-edge SXE spectra plotted as a function of loss energy. Inset: Ti $L$-edge XAS spectrum with markers indicating excitation energies used in this series of emission spectra. The uppermost emission spectrum in panel (a) was obtained with a photon energy of $480 \mathrm{eV}$. The triangular markers in the emission spectra indicate the excitation energy used for each spectrum. The emission spectra are vertically offset for clarity with the interval being proportional to the difference in excitation energies between successive spectra. The vertical lines are a guide to the eye to indicate the location of PDOS features (a) or loss and RIXS features (b). $\sim 460.5 \mathrm{eV}$ is a transition to an $e_{g}^{*}$ final state. Similarly the peaks at $463.6 \mathrm{eV}$ and $466.1 \mathrm{eV}$ are $2 p_{1 / 2}$ transitions to $t_{2 g}^{*}$ and $e_{g}^{*}$ states, respectively. The splitting between the $t_{2 g}^{*}$ and $e_{g}^{*}$ states is in each case $\sim 2.4 \mathrm{eV}$. Although in general the energy splitting in the XAS spectra is not equal to the crystal-field parameter $10 D q$, by reference to the model calculations for $\mathrm{Ti}^{4+}$ in $O_{h}$ symmetry of De Groot et al., an estimate can be made for the parameter $10 D q$ of, in fact, $2.4 \mathrm{eV} .{ }^{20}$ In contrast to the published Ti $L$-edge XAS spectra of $\mathrm{SrTiO}_{3}, \mathrm{BaTiO}_{3}$, and $\mathrm{Sr}_{0.5} \mathrm{Ba}_{0.5} \mathrm{TiO}_{3},{ }^{18}$ it can be seen that the $e_{g}^{*}$ peak at $\sim 460.5 \mathrm{eV}$ is both broadened and reduced in intensity in comparison to the lower-energy $t_{2 g}^{*}$ peak and clearly displays spectral features with components at energies of $\sim 460.2$ and $\sim 460.9 \mathrm{eV}$. There is a lowered symmetry of the $\mathrm{Ti}^{4+}$ in CCTO in comparison to the octahedral $O_{h}$ point-group symmetry in the aforementioned perovskites. In CCTO the octahedron around the $\mathrm{Ti}^{4+}$ ion is tilted with respect to the axis of the cubic cell by an angle of $19.5^{\circ}$ with alternate octahedra in the $x-y$ plane canted in differing directions as is clearly seen in Fig. 1, and the new point-group symmetry of the $\mathrm{Ti}^{4+}$ site is $C_{3 i}$. The $e_{g}$ orbitals of $\mathrm{Ti}$ are oriented towards the ligands in a $\mathrm{TiO}^{6}$ octahedron whereas the $t_{2 g}$ 's are oriented towards the space between the ligands. As a result the broadening of the $e_{g}^{*}$ feature comes from the $e_{g}$ states being more strongly coupled to the lattice than are the $t_{2 g}$ states. It has been observed that single-crystal samples of CCTO are mainly twinned in nanoscale domains which differ in the sign of rotation of the $\mathrm{TiO}_{6}$ octahedra. ${ }^{1}$ The measured giant dielectric effect may thus have an "extrinsic" effect due to an internal barrier layer capacitance between these twinned regions. ${ }^{1,6}$

In contrast to the complex absorption spectra, the SXE spectrum in Fig. 3 closely reflects the PDOS of the occupied valence band states of $\mathrm{Ti} 3 d$ character. Here the excitation energy of $460.9 \mathrm{eV}$ was chosen such that only Ti $2 p_{3 / 2}$ states are ionized. This avoids recording overlapping emission due to $\mathrm{Ti} 3 d$ electrons making transitions into both spin-orbit split $\mathrm{Ti} 2 p$ core levels and simplifies the comparison to theory. However, at this energy, elastic and inelastic scattering in the SXE spectrum is significant, and in addition to simple PDOS fluorescence, the spectrum contains components due to resonant inelastic x-ray scattering (RIXS) as will be discussed below. In RIXS, the incident photon, in resonance with a core absorption, scatters from the system, losing energy to a valence excitation, and exits with a reduced energy. RIXS features can overlap with PDOS fluorescence features, and this makes comparison to theory more challenging since typical electronic-structure calculations of the PDOS will not include RIXS excitations.

Figure 4 shows a series of SXE spectra recorded as a function of incident photon energy. The photon energies used are indicated on the XAS spectrum included as an inset in 
Fig. 4. As the incident photon energy is varied, two types of emission features can be identified: features that appear at a constant emission energy, independent of changes in the incident energy, and features whose emission energy changes at a constant offset or energy loss from the incident photon energy as the latter is varied. The first type is conventional PDOS features, which appear at a constant emission energy since the energy difference between the valence and core states is, to a first approximation, independent of the excitation energy. The second type of emission feature is due to excitations of electrons in the valence band and is best described in terms of RIXS. To more clearly distinguish between these two types of emission features, the measured SXE spectra are plotted both against emission energy [Fig. 4(a)] and against energy loss with respect to the excitation energy [Fig. 4(b)]. The PDOS features of the Ti $L_{2,3}$ SXE spectra can be broadly grouped into a Ti $L_{\alpha}$ emission feature centered at an emission energy of approximately $451 \mathrm{eV}$ and a Ti $L_{\beta 1}$ emission feature centered at an emission energy of approximately $458 \mathrm{eV}$ as indicated in Fig. 4(a). The SXE spectrum shown in Fig. 3 is the fifth from the bottom in the series presented in Fig. 4(a) and is the last emission spectrum before the $L_{2}$ edge that does not have components due to excitation of the Ti $2 p_{1 / 2} \rightarrow 3 d$ multiplet. Furthermore, the principal $L_{\alpha}$ component of this spectrum is least affected by overlapping RIXS features. Thus this experimental spectrum can most easily be compared to a calculated SXE spectrum derived from the Ti $3 d$ valence-band PDOS.

A model SXE spectrum based upon the calculated Ti $3 d$ PDOS broadened by the core-hole lifetime, the valence-hole lifetime, and instrumental broadening is also depicted on the left in the lower part of Fig. 3. For the purposes of visual comparison and to align the model spectrum with the measured spectrum, the Ti $3 d$ conduction-band PDOS, broadened by both core-hole lifetime and instrumental broadening, is also shown on the right in the lower part of Fig. 3. The energy scales between experiment and calculation are aligned by the $t_{2 g}^{*}$ peak in the conduction band. The broadened conduction band PDOS does not take into account the $L_{2}$ edge, since as previously noted an atomic multiplet model is a more accurate way to model the Ti $L_{2,3}$ XAS spectrum and thus a direct comparison with the present calculation virtual conduction-band PDOS is unrealistic. ${ }^{19,20}$ However, comparing the measured SXE spectrum at this excitation energy with the model SXE spectrum reveals close agreement between theory and experiment, although it is clear that the valence-band width is underestimated in the calculations. The high-energy part of this spectrum most likely is due to resonant fluorescence. There is of course overlap in energy of the Ti $3 d$ states with the O $2 p$ states where the hybridization is largely confined to the lower part of the valence band. Finally, it should be noted that there is no evidence in the Ti-edge XAS spectrum of the low-lying peak in the conduction-band virtual PDOS states which is primarily due to spin-down $\mathrm{Cu} 3 d_{x y}$ states. Any such signature in the Ti XAS requires the $\mathrm{Cu}$ state to have a presence on the Ti site, and although the site-projected local PDOS shown in Fig. 2 appears to indicate that this is the case, there is another already documented reason for the absence of such a feature. This absence is a consequence of the failure of this and of earlier calculations to accurately give the gap of this material where the on-site $\mathrm{Cu}$ Coulomb exchange integral $U_{d d}$ must be underestimated and thus the splitting between the $\mathrm{Cu} 3 d_{x y}$ states must be larger than anticipated. ${ }^{14}$

It should be noted that two new features can be seen in the spectrum obtained at the highest excitation energy shown in Fig. 4(a). The excitation energy used for this spectrum was $\sim 480 \mathrm{eV}$. These features are at seen at emission energies of $\sim 465.2 \mathrm{eV}$ and $475.0 \mathrm{eV}$ and are not $\mathrm{Ti}$ emission features but are rather $\mathrm{Cu} L_{\alpha}$ and $L_{\beta 1}$ emission features observed in second-order emission and excited by second-order light from the monochromator.

We now consider the RIXS features present in the Ti emission spectra. As noted earlier, valence-band excitation RIXS features are distinguishable from simple DOS-related fluorescence features in emission spectra by the fact that the RIXS features appear at a constant loss energy relative to the excitation energy, since the incident beam is losing energy to a valence excitation. In contrast, fluorescent features appear at a constant emission energy since they are related to valence-state to core-state transitions. Thus, if features appear in emission spectra that change their energy in step with changes in the excitation energy, they are identified as RIXS features. Figure 4(b) plots the same data set from Fig. 4(a), but on an energy loss scale relative to the incident photon energy. Several possible RIXS features that persist at a constant energy loss from the excitation energy are visible in Fig. 4(b). These occur at loss energies of approximately 7.0, $5.5,4.3$, and $0.9 \mathrm{eV}$ and are marked $A, B, C$, and $D$, respectively in Fig. 4(b). Feature $A$ is the RIXS feature with the largest intensity, resonating when the excitation energy is near the $L_{3}$ edge (bottom two spectra) and near the $L_{2}$ edge (sixth and seventh from bottom). The photon energy dependence of the Ti $L$-edge emission spectra in Fig. 4 is very similar to that measured from the perovskites $\mathrm{Ba}_{0.5} \mathrm{Sr}_{0.5} \mathrm{TiO}_{3},{ }^{18} \mathrm{BaTiO}_{3},{ }^{11}$ and in the nonperovskite $\mathrm{FeTiO}_{3},{ }^{21}$ but differs from that measured in rutile $\mathrm{TiO}_{2} \cdot{ }^{22}$ Uehara et al. identify the primary RIXS excitation (feature $A$ ) as being due to a charge-transfer (CT) excitation where the incident photon scatters from the valence electrons, exciting an $\mathrm{O} 2 p$ valence electron into an unoccupied Ti $3 d$ state. Thus the final state of the scattering event is an electron-hole pair or valence exciton which is a nonbonding $3 d^{1} L$ state where $L$ is a ligand (O $2 p$ ) valence hole. ${ }^{18,21} \mathrm{~A}$ precise determination of the energy of this CT excitation is difficult due to the large width of the hybridized O $2 p / \mathrm{Ti} 3 d$ valence band. Features $B$ and $C$ appear to be fairly sharp features, whereas in general it is expected that Raman scattering features should be broad, and further they are not clearly visible in all spectra. Finally, feature $D$, which appears to be a RIXS feature with a very low loss energy of $\sim 1 \mathrm{eV}$, is present in all spectra from the third from bottom upwards in Fig. 4(b) (note that in the two uppermost spectra feature $D$ overlaps partly with the $\mathrm{Cu} L_{\alpha}$ emission). The exact origin of these RIXS features has yet to be determined. Finally, at a zero loss energy, coincident with the excitation energy, is the elastically scattered or recombination peak and it displays significant changes in intensity as the incident photon energy goes in and out of resonance with features in the unoccupied PDOS and in particular is enhanced at both 


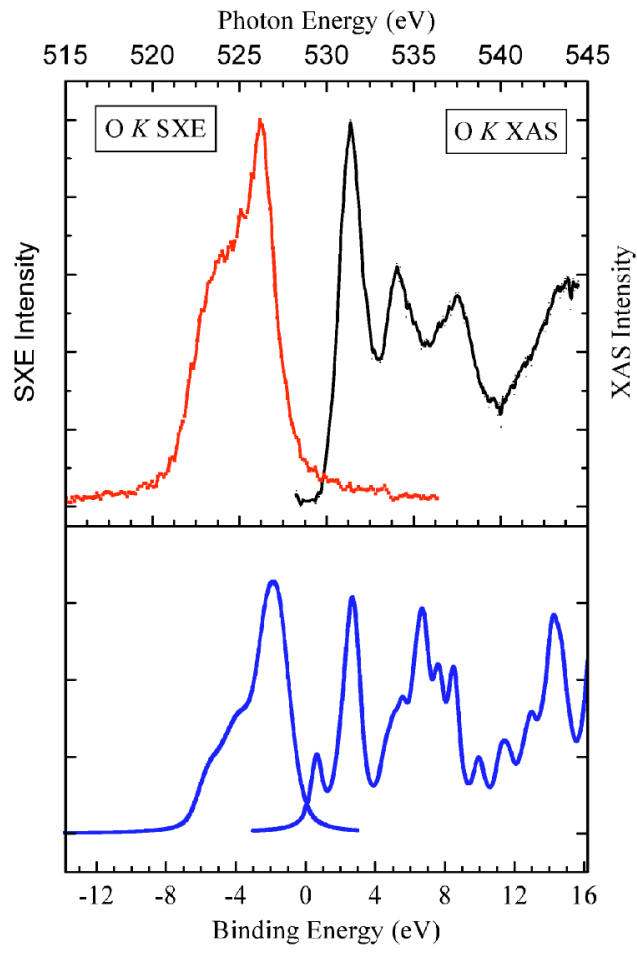

FIG. 5. O $K$-edge XAS and SXE spectra from CCTO are shown in the upper panel. The XAS spectrum on the right of the figure corresponds to absorption from the $\mathrm{O} 1 s$ state. The SXE spectrum on the left was recorded with an excitation energy of $551.2 \mathrm{eV}$ and corresponds to radiative transitions into holes on the $\mathrm{O} 1 s$ level. The lower panel shows the calculated O $K$ SXE and XAS on the left and right, respectively. The calculated spectra are derived from the $\mathrm{O} 2 p$ PDOS and broadened by the core-hole lifetime and instrumental broadening. Experimental and theoretical energy scales are aligned by reference to the $t_{2 g}$ peak in the XAS spectrum.

the $L_{3}$ and $L_{2}$ edges. Both Uehara et al. and Butorin et al. note the resonant enhancement of this feature, as well as the resonant enhancement of feature $A$, the CT excitation, at both the $L_{3}$ and $L_{2}$ edges. A more comprehensive discussion of $\mathrm{Ti}$ $L_{2,3}$ RIXS in nominally $d^{0}$ compounds such as CCTO can be found in Butorin et al. ${ }^{21}$ In their analysis of such scattering in $\mathrm{FeTiO}_{3}$ it was estimated that the onset of continuum states in $\mathrm{FeTiO}_{3}$, above which nonresonant normal fluorescence occurs, is at an energy of about $1 \mathrm{eV}$ above the $t_{2 g}$ peak in the $L_{3}$ absorption edge. This estimate is based on the combination of Ti $2 p$ photoemission, valence-band photoemission, and optical spectroscopies, not all of which are currently available for CCTO. However, it is reasonable to assume that CCTO should be similar, and thus the Ti SXE spectrum in Fig. 3 will be dominated by the normal PDOS fluorescence.

\section{B. Oxygen states}

The $\mathrm{O} K$-edge SXE and XAS spectra corresponding to the occupied and unoccupied O $2 p$ PDOS are presented in Fig. 5 . The XAS spectrum on the right of the figure results from the absorption of electrons from the $\mathrm{O} 1 s$ core level into unoccupied conduction-band states of primarily $\mathrm{O} 2 p$ character. The SXE spectrum on the left of the figure was re- corded with an incident photon energy of $551.2 \mathrm{eV}$, well above the absorption threshold. The SXE spectrum is a direct measure of the PDOS of the occupied O $2 p$ states. Unlike the case for the Ti $L_{2,3}$ edge the O $K$-edge XAS (and SXE) spectra can be directly compared to the O $2 p$ PDOS calculated through single-particle schemes ${ }^{23}$ although strictly speaking a DFT calculation gives empty virtual conductionband states. Thus in the lower half of Fig. 5 we present the $\mathrm{O}$ $K$-edge SXE and XAS spectra based upon the calculated O $2 p$ PDOS where these are broadened by the appropriate corehole and valence lifetimes and by the instrumental function.

The O $K$-edge XAS spectrum for CCTO is similar to that of the perovskite $\mathrm{SrTiO}_{3},{ }^{23}$ with three discrete absorption features visible within $10 \mathrm{eV}$ of the $\mathrm{O} K$-edge absorption. For CCTO, these occur at photon energies of $531.4 \mathrm{eV}$, $534.0 \mathrm{eV}$, and $537.5 \mathrm{eV}$, with a separation between the first two peaks, which correspond to the $t_{2 g}^{*}$ and $e_{g}^{*}$ states, of $2.6 \mathrm{eV}$. This compares to $\mathrm{SrTiO}_{3}$, where the separation is approximately $2.4 \mathrm{eV}$, while the intensity ratio and shape of these features are very similar. In $\mathrm{SrTiO}_{3}$ the features observed immediately above the $e_{g}^{*}$ peak are due to Sr states, while in CCTO the single peak at $537.5 \mathrm{eV}$ can be attributed, by comparison with the calculated PDOS, as being due to $\mathrm{Ca}$ $3 d$ states. This peak is observed at $6.1 \mathrm{eV}$ above the $t_{2 g}^{*}$ peak, but the calculated $\mathrm{Ca} 3 d$ PDOS peak lies at an energy of $6.7 \mathrm{eV}$, only $4.0 \mathrm{eV}$ above the $t_{2 g}^{*}$ peak coinciding with the high-energy side of the calculated position of the $e_{g}^{*}$ peak. The measured unoccupied O $2 p$ PDOS differs from the DFT calculation presented in Fig. 2 and here in Fig. 5 inasmuch as the predicted $\mathrm{O} 2 p$ PDOS feature just above threshold is not seen. The absence of this feature was previously noted in connection with the Ti $L_{2,3}$ XAS. However, other than this there is good agreement between theory and experiment for

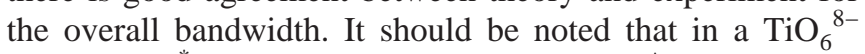
cluster, the $t_{2 g}^{*}$ orbitals normally give rise to $\pi^{*}$ states and the $e_{g}^{*}$ orbitals give rise to $\sigma^{*}$ states. The model O $K$-edge SXE spectrum derived from the valence-band $\mathrm{O} 2 p$ states shown at the lower left in Fig. 5 is calculated to be emission from $\pi$ states in the valence band.

Figure 6 presents a series of O $K$-edge SXE spectra recorded as a function of incident photon energy, with the excitation energy indicated on the XAS spectrum presented on the inset. While some changes in the shape of the SXE spectra can be observed, there is no evidence of any RIXS excitations, in contrast to the Ti $L$-edge spectra presented in Fig. 4. Rather the change in shape of the SXE spectra is due to the fact that at threshold the excitation energy is initially coincident with the $\pi^{*}$ states in the conduction band and when increased coincides with the $\sigma^{*}$ states. As is well known for resonant SXE from molecular systems, in a $90^{\circ}$ measurement geometry, when exciting $\sigma^{*}$ states, the resultant measured emission is from $\pi$ states and vice versa. ${ }^{24} \mathrm{In}$ CCTO the oxygen atoms are surrounded by three metal atoms (two Ti and one $\mathrm{Cu}$ ), forming an $M_{3} \mathrm{O}$ cluster with point group $C_{s}$. This local coordination of the oxygen atom can be seen clearly in Fig. 1. The $M_{3} \mathrm{O}$ cluster is almost perfectly planar with two identical bond angles of $109^{\circ}$ and one of $141^{\circ}$, leading to an $s p^{2}$ hybridization of the central $\mathrm{O}$ to form three $\sigma$ bonds and one $\pi$ bond perpendicular to the plane. Thus in the lower part of Fig. 6 we present separately calcu- 


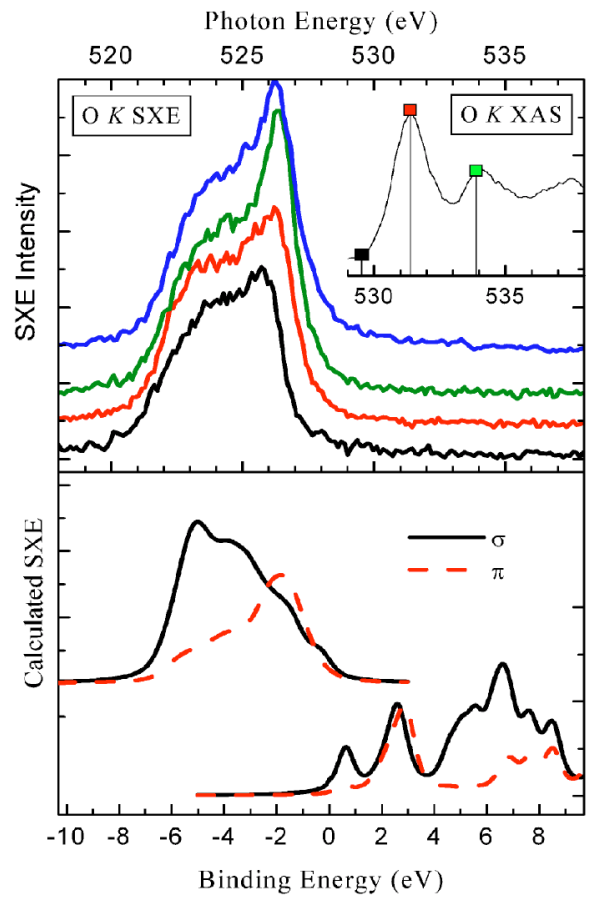

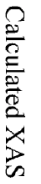

FIG. 6. Series of O $K$-edge SXE spectra as a function of excitation energy. The photon energies used are indicated by the markers and vertical lines in the XAS spectrum included in the upper left. The topmost SXE spectrum is that shown in Fig. 2 with an excitation energy of $551.2 \mathrm{eV}$. The SXE spectra are vertically offset for clarity. The lower panel shows the calculated O $2 p$ SXE from $\pi$ and $\sigma$ states as well as the calculated XAS spectrum to $\pi^{*}$ and $\sigma^{*}$ states. Experimental and theoretical energy scales are aligned by reference to the $t_{2 g}$ peak in the XAS spectrum.

lations for the valence-band emission from $\pi$ states and from $\sigma$ states. Also presented in the lower part of Fig. 6 are the $\pi^{*}$ and $\sigma^{*}$ components in the conduction band. Hence at excitation energies at threshold and at the first absorption peak at $531.4 \mathrm{eV}$ there is a mixture of $\sigma$ - and $\pi$-state emission $\left(\pi^{*}\right.$ and $\sigma^{*}$ absorption). At higher energies it is emission from $\pi$ states ( $\sigma^{*}$ absorption) that tends to dominate. The O $K$-edge SXE spectra thus agree very well with these calculated O $2 p$ PDOS, particularly for excitation far above threshold. Although the full width at half maximum (FWHM) of $\sim 5.2 \mathrm{eV}$ of the calculated emission is in good agreement with the measurement, the peak of the x-ray emission appears to be farther away in energy from the $t_{2 g}$ peak in the XAS spectrum than the equivalent energy separation in the calculation. The peak in the calculated PDOS for the $\pi$ states is coincident with the peak in the $\mathrm{Cu} 3 d$ PDOS and corresponds to strong hybridization between them. A greater value of the on-site $\mathrm{Cu} U_{d d}$ may result in this peak moving to lower energies in the calculated spectra. The occupied component of the $\mathrm{O} 2 p$ PDOS which is closest to the valence-band maximum is that which is hybridized with the $\mathrm{Cu} 3 d_{x y}$ orbital. These states in fact hybridize with the $\mathrm{O} 2 p \sigma$ states which are directed toward the $\mathrm{Cu}$ atom. Thus, if these predicted $\mathrm{Cu}$ $3 d_{x y}$ features close to and on either side of $E_{\mathrm{F}}$ were more greatly separated, then the conduction-band feature would move to higher energies and become part of the first observed peaks in both the Ti $L_{2,3}$ and $\mathrm{O} K$ XAS while the
Photon Energy (eV)

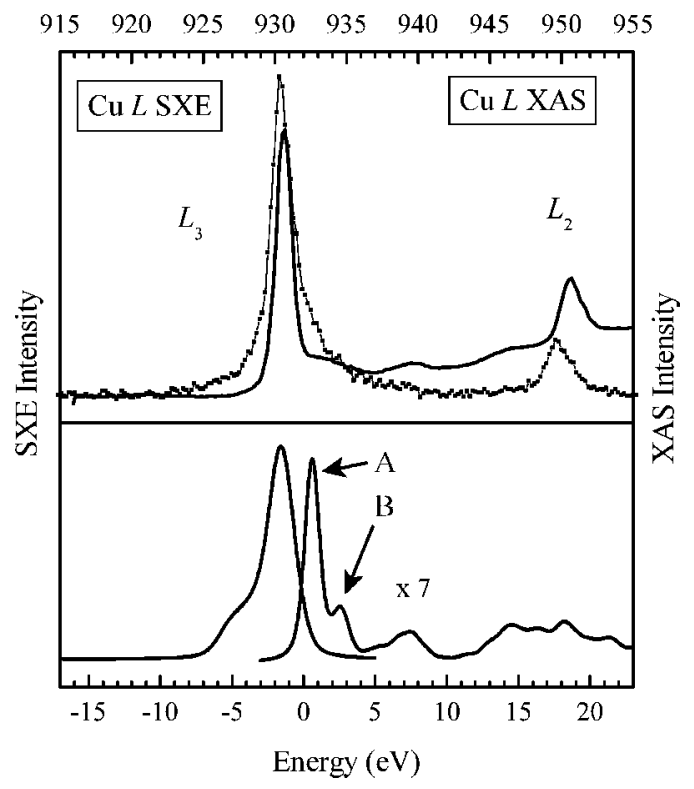

FIG. 7. $\mathrm{Cu} L$-edge XAS and SXE spectra from CCTO. The XAS spectrum (solid) to the right of the figure corresponds to absorption from the $\mathrm{Cu} 2 p_{1 / 2}$ and $2 p_{3 / 2}$ states. The SXE spectrum (dots) to the left was recorded with an excitation energy of $965 \mathrm{eV}$. The lower half of the figure shows the calculated $\mathrm{Cu} L_{\alpha}$ SXE and XAS spectra based on the $\mathrm{Cu} 3 d$ PDOS taking into account core-hole lifetime and instrumental broadening. The intensity of the calculated XAS spectrum is multiplied by 7 with respect to the calculated SXE spectrum for ease of comparison with the measured spectra.

valence-band feature would move to lower energies. The occupied $\mathrm{O} 2 p \sigma$ states would then have almost no component close to the valence-band maximum and the observed shift in the emission edge on going from threshold excitation at $529.5 \mathrm{eV}$ to excitation at $531.4 \mathrm{eV}$ would be well reproduced.

\section{Copper states}

Figure 7 presents $\mathrm{Cu} L$-edge XAS and SXE spectra. The XAS spectrum on the right of the figure results from the excitation of electrons from both the $\mathrm{Cu} 2 p_{1 / 2}$ and $\mathrm{Cu} 2 p_{3 / 2}$ states into unoccupied $\mathrm{Cu} 3 d$ states. The SXE spectrum was recorded with an incident photon energy of $965 \mathrm{eV}$. The SXE spectrum results from the deexcitation of electrons with primarily $\mathrm{Cu} 3 d$ character into the $\mathrm{Cu} 2 p_{3 / 2}\left(L_{3}\right.$ edge) or $\mathrm{Cu}$ $2 p_{1 / 2}\left(L_{2}\right.$ edge) hole, both of which can be seen in the spectrum. For the purposes of comparison the lower half of Fig. 7 depicts the calculated $L_{\alpha}$ SXE and XAS spectra which are based on the $\mathrm{Cu} 3 d$ PDOS shown in Fig. 2.

The $\mathrm{Cu} L_{2,3}$ XAS spectrum for CCTO is similar to that measured in other $\mathrm{CuO}$ compounds such as $\mathrm{La}_{1.85} \mathrm{Sr}_{0.15} \mathrm{CuO}_{4},{ }^{25}$ but differs somewhat from simple $\mathrm{CuO} .{ }^{26}$ The energy scale shown for the XAS spectrum was determined from comparing to $\mathrm{Cu} L$-edge XAS of $\mathrm{Cu}$ metal and the error in energy is estimated to be $\pm 0.5 \mathrm{eV}$. The XAS spectrum was measured in TEY mode and following background correction is very similar to that obtained in TFY 
mode (not shown). A number of discrete absorption features are visible. The two most intense features, at $930.7 \mathrm{eV}$ and $950.6 \mathrm{eV}$, result from absorption from the spin-orbit split $\mathrm{Cu}$ $2 p_{3 / 2}$ and $2 p_{1 / 2}$ states-i.e., the $L_{3}$ and $L_{2}$ edges. In each case almost all the intensity in the near-edge region is concentrated in a narrow peak at an energy lower than the XPS threshold. ${ }^{26}$ This shift of the XAS threshold to lower energies is the result of core-hole effects in such cuprates resulting in an excitonic peak immediately below the conduction-band minimum. The information concerning the unoccupied $\mathrm{Cu}$ $3 d$ PDOS is contained in the weak structures that follow these excitonic peaks, though this information may be masked by polaronic effects, ${ }^{27}$ as well as charge-transfer satellites. The $\mathrm{Cu} L_{2,3}$ XAS spectra is a good indicator of the $\mathrm{Cu}$ valence and in this case appears to indicate a $\mathrm{Cu}$ (II) compound as would be anticipated. ${ }^{26}$ The energy difference between the two spin-orbit split excitonic features in the XAS spectrum is $19.9 \mathrm{eV}$ and is the separation of the $\mathrm{Cu} 2 p_{3 / 2}$ and $2 p_{1 / 2}$ states, which is in good agreement with XPS measurements of the $\mathrm{Cu} 2 p$ spin-orbit core-level splitting of $20 \mathrm{eV}$ in $\mathrm{CuO}^{28}$ Comparing to the calculated XAS spectrum based on the $\mathrm{Cu} 3 d$ PDOS, it can be seen that two features labeled $A$ and $B$ are predicted in the absorption spectrum. In this instance the energy scales of the calculated and experimental spectra are set by aligning the emission peak in the respective spectra. Note that both the larger $(A)$ and the smaller $(B)$ calculated XAS features are assigned to $\mathrm{Cu} 3 d_{x y}$ states where these orbitals are in the plane of the $\mathrm{CuO}_{4}$ plaquette pictured in Fig. 1 and the copper atoms have point group $D_{2 h}$. The position in energy of the larger peak $(A)$, assigned to spindown $\mathrm{Cu} 3 d_{x y}$ states immediately above $E_{\mathrm{F}}$, has previously been questioned (see above). This question is not resolved here, since only a single feature following the excitonic peak, at about $933.7 \mathrm{eV}$, is discernible in the measured XAS spectrum. The separations between the calculated peak in the emission spectrum and the calculated peaks in the absorption are $\sim 2.1 \mathrm{eV}$ and $\sim 4.1 \mathrm{eV}$ for features $A$ and $B$ in the conduction band, respectively.

Finally, the SXE spectrum in Fig. 7 does not necessarily reflect the PDOS of the occupied states of $\mathrm{Cu} 3 d$ character in the valence band. At the excitation energy of $965 \mathrm{eV}$, holes are created on both $\mathrm{Cu} 2 p_{3 / 2}$ and $2 p_{1 / 2}$ states. The peak in the observed $L_{\alpha}$ emission is at $\sim 930.3 \mathrm{eV}$ and is measured in second order with an effective energy resolution of $1.3 \mathrm{eV}$. Thus there is a separation of $\sim 3.2 \mathrm{eV}$ between the peak in the emission and the possible PDOS-associated peak in the absorption spectra, which would is in good agreement with calculations. Considering the SXE spectrum in detail, in common with other $\mathrm{Cu}-\mathrm{O}$ charge-transfer systems, it can be assumed that there is one hole shared between the $\mathrm{Cu} 3 d$ and $\mathrm{O} 2 p$ states. This state is then a linear superposition of the $3 d^{9}$ and $3 d^{10} \underline{L}$ configurations. In the case of the $\mathrm{Cu} L_{\alpha}$ spectrum, due to core ionization, the valence electron moves from the ligand atom to the $\mathrm{Cu}$ atom. Thus the dominant transition should be $\mathrm{Cu} 2 p d^{10} \underline{L} \rightarrow d^{9} \underline{L}$ for the $\mathrm{Cu} L_{\alpha}$ line. ${ }^{29}$

\section{CONCLUSIONS}

First the experimental results of this X-ray spectroscopic study of $\mathrm{CaCu}_{3} \mathrm{Ti}_{4} \mathrm{O}_{12}$ are in close agreement with the theoretical results presented both here and elsewhere. ${ }^{14} \mathrm{The} \mathrm{Ti}$ $L_{2,3}$-edge XAS spectra reflect the reduced symmetry of the central $\mathrm{Ti}$ ion as compared to the other perovskite compounds $\mathrm{BaTiO}_{3}$ and $\mathrm{SrTiO}_{3} \cdot{ }^{18} \mathrm{Ti} L_{\alpha, \beta} \mathrm{SXE}$ spectra at and above the $L_{3}$ and $L_{2}$ edges show similar RIXS features and resonant behavior as other $\mathrm{Ti} d^{0}$ perovskites such as $\mathrm{Ba}_{0.5} \mathrm{Sr}_{0.5} \mathrm{TiO}_{3},{ }^{18}$ as well as nonperovskites such as $\mathrm{FeTiO}_{3} \cdot{ }^{21}$ The principal RIXS excitation observed is a charge-transfer excitation to a nonbonding Ti $3 d^{1} \mathrm{O} 2 p^{-1}$ state. O $K$-edge SXE spectra are in excellent agreement with calculations, particularly as the resonant behavior of the O $K$ SXE spectrum can be explained in terms of $\sigma$ - and $\pi$-state emission from valence-band $\mathrm{O} 2 p$ states when in resonance with $\pi^{*}$ and $\sigma^{*}$ conduction-band $\mathrm{O} 2 p$ states. Comparison of $\mathrm{O} K$ XAS spectra with calculations indicates that the calculated position of the $\mathrm{Ca} 3 d$ states in the conduction band is at least $2 \mathrm{eV}$ lower than is observed. Furthermore, a consistent problem in both this and previous calculations is the treatment of the $\mathrm{Cu} 3 d_{x y}$ states at the center of the $\mathrm{CuO}_{4}$ plaquette. The experimental results presented here, as well as the measured band gap of the material, do not indicate that these states are to be found immediately above $E_{\mathrm{F}}$ and a more thorough analysis of how to account for this in calculations is needed.

\section{ACKNOWLEDGMENTS}

The Boston University program is supported in part by the Department of Energy under Grant No. DE-FG0298ER45680. The $\mathrm{x}$-ray emission spectrometer system was funded by the U.S. Army Research Office under Grant Nos. DAAD19-01-1-0364 and DAAH04-95-0014. The experiments at the NSLS are supported by the U.S. Department of Energy, Division of Materials and Chemical Sciences. P.S. acknowledges the support of the William V. Shannon Memorial Fellowship. C.McG. wishes to acknowledge financial support from the Irish Higher Educational Authority.

\footnotetext{
*Present address: School of Chemical and Physical Sciences, Victoria University of Wellington, Wellington, New Zealand.

Present address: Department of Physics, University College Dublin, Belfield, Dublin 4, Ireland.

Corresponding author. Electronic address: ksmith@bu.edu

${ }^{1}$ M. A. Subramanian, D. Li, N. Duan, B. A. Reisner, and A. W. Sleight, J. Solid State Chem. 151, 323 (2000).
}

${ }^{2}$ A. P. Ramirez, M. A. Subramanian, M. Gardel, G. Blumberg, D. Li, T. Vogt, and S. M. Shapiro, Solid State Commun. 115, 217 (2000).

${ }^{3}$ C. C. Homes, T. Vogt, S. M. Shapiro, S. Wakimoto, and A. P. Ramirez, Science 293, 673 (2001).

${ }^{4}$ W. Si, E. M. Cruz, P. D. Johnson, P. W. Barnes, P. Woodward, and A. P. Ramirez, Appl. Phys. Lett. 81, 2056 (2002). 
${ }^{5}$ C. C. Homes, T. Vogt, S. M. Shapiro, S. Wakimoto, M. A. Subramanian, and A. P. Ramirez, Phys. Rev. B 67, 092106 (2003).

${ }^{6}$ D. C. Sinclair, T. B. Adams, F. D. Morrison, and A. R. West, Appl. Phys. Lett. 80, 2153 (2002).

${ }^{7}$ J. Nordgren, G. Bray, S. Cramm, R. Nyholm, J. E. Rubensson, and N. Wassdahl, Rev. Sci. Instrum. 60, 1690 (1989).

${ }^{8}$ C. McGuinness, G. Hughes, J. Roche, D. Fu, J. Downes, and K. E. Smith, J. Appl. Phys. 94, 3919 (2003).

${ }^{9}$ J. Nordgren and R. Nyholm, Nucl. Instrum. Methods Phys. Res. A 246, 242 (1986).

${ }^{10}$ R. Brydson, H. Sauer, W. Engel, J. M. Thomas, E. Zeitler, N. Kosugi, and H. Kuroda, J. Phys.: Condens. Matter 1, 797 (1989); R. Brydson, H. Sauer, W. Engel, and F. Hofer, ibid. 4, 3429 (1992).

${ }^{11}$ J. Jimenez-Mier, J. van Ek, D. L. Ederer, T. A. Callcott, J. J. Jia, J. Carlisle, L. Terminello, A. Asfaw, and R. C. Perera, Phys. Rev. B 59, 2649 (1999).

${ }^{12}$ J. Jimenez-Mier, U. Diebold, D. L. Ederer, T. A. Callcott, M. Grush, and R. C. Perera, Phys. Rev. B 65, 184105 (2002).

${ }^{13}$ B. L. Henke, E. M. Gullikson, and J. C. Davis, At. Data Nucl. Data Tables 54, 181 (1993).

${ }^{14}$ L. X. He, J. B. Neaton, M. H. Cohen, D. Vanderbilt, and C. C. Homes, Phys. Rev. B 65, 214112 (2002).

${ }^{15}$ P. Blaha, K. Schwarz, G. K. H. Madsen, D. Kvasnicka, and J. Luitz, WIEN2k, an augmented plane wave+local orbitals program for calculating crystal properties, Karlheinz Schwarz, Technical Universität Wien, Austria, 2001.

${ }^{16}$ J. P. Perdew, K. Burke, and M. Ernzerhof, Phys. Rev. Lett. 77, 3865 (1996).

${ }^{17}$ S. Kumar, V. S. Raju, and T. R. N. Kutty, Appl. Surf. Sci. 206,
250 (2003).

${ }^{18}$ Y. Uehara, D. W. Lindle, T. A. Callcott, L. T. Terminello, F. J. Himpsel, D. L. Ederer, J. H. Underwood, E. M. Gullikson, and R. C. C. Perera, Appl. Phys. A: Mater. Sci. Process. 65, 179 (1997).

${ }^{19}$ F. M. F. de Groot, J. C. Fuggle, B. T. Thole, and G. A. Sawatzky, Phys. Rev. B 42, 5459 (1990).

${ }^{20}$ F. M. F. de Groot, J. C. Fuggle, B. T. Thole, and G. A. Sawatzky, Phys. Rev. B 41, 928 (1990).

${ }^{21}$ S. M. Butorin, J. H. Guo, M. Magnuson, and J. Nordgren, Phys. Rev. B 55, 4242 (1997).

${ }^{22}$ Y. Tezuka, S. Shin, A. Agui, M. Fujisawa, and T. Ishii, J. Phys. Soc. Jpn. 65, 312 (1996); L. D. Finkelstein, E. Z. Kurmaev, M. A. Korotin, A. Moewes, B. Schneider, S. M. Butorin, J. H. Guo, J. Nordgren, D. Hartmann, M. Neumann, and D. L. Ederer, Phys. Rev. B 60, 2212 (1999).

${ }^{23}$ F. M. F. de Groot, J. Faber, J. J. M. Michiels, M. T. Czyzyk, M. Abbate, and J. C. Fuggle, Phys. Rev. B 48, 2074 (1993).

${ }^{24}$ F. Gel'mukhanov and H. Agren, Phys. Rep. 312, 91 (1999).

${ }^{25}$ C. T. Chen, L. H. Tjeng, J. Kwo, H. L. Kao, P. Rudolf, F. Sette, and R. M. Fleming, Phys. Rev. Lett. 68, 2543 (1992).

${ }^{26}$ M. Grioni, J. B. Goedkoop, R. Schoorl, F. M. F. de Groot, J. C. Fuggle, F. Schaefers, and E. E. Koch, Phys. Rev. B 39, 1541 (1989).

${ }^{27}$ G. van der Laan, Phys. Rev. B 41, 12366 (1990).

${ }^{28}$ K. Allan, A. Campion, J. Zhou, and J. B. Goodenough, Phys. Rev. B 41, 11572 (1990).

${ }^{29}$ J. A. Leiro, F. Werfel, and G. Drager, Phys. Rev. B 44, 7718 (1991). 